\title{
Design of Pyramid Column Sensor with Photodiodes for Measuring Solar Power
}

\author{
Yong-Nong Chang* and Hung-Liang Cheng ${ }^{1}$ \\ Department of Electrical Engineering, National Formosa University, \\ No. 64, Wunhua Rd., Huwei Township, Yunlin County 63201, Taiwan \\ 'Department of Electrical Engineering, I-Shou University, \\ Kaohsiung, No.1, Sec. 1, Syuecheng Rd., Dashu District, Kaohsiung City 84001, Taiwan
}

(Received December 24, 2013; accepted March 5, 2014)

Key words: photodiode detector, solar power, pyramid column sensor, coordinate transformation

In this study, five photodiode detectors are used to constitute a solar power sensor that can measure both the directly incident and undisciplined power of solar light. The solar power sensor is composed of four trapezoidal facets along with an upper rectangular facet formed on top to constitute a rectangular pyramid column sensor. On each facet, a photodiode sensor is implemented to collect solar light power coming from different orientations including the directly incident and undisciplined power. By applying a perpetual calendar to obtain the corresponding azimuth angle and elevation angle of sunlight, the collected power data of the five detectors are employed to estimate the maximum directly incident power and undisciplined power of solar light by simple geometric transformation. Therefore, the proposed rectangular pyramid column sensor can effectively provide the detailed solar power data including directly incident and undisciplined power.

\section{Introduction}

The endless usage of fossil energy has led to problems such as energy shortage and the greenhouse effect. Solar power, which is clean, environment friendly, and sustaining in nature, plays a crucial role in harnessing green energy. ${ }^{(1)}$ For the effective use of solar power in heating and power generation, precise estimation of the collected solar power is indispensable..$^{(2,3)}$ Taking a solar energy plant as an example, the generating capacity is related to not only the incident solar energy itself, but also the efficiency of the solar panel and the mutual orientation relationship between the solar panel and sunlight. The collected power is converted and interconnected with a power system by a photovoltaic inverter. Hence, the correct solar power information, such as light orientation and solar power, are the prerequisites of an efficient solar power system. ${ }^{(4,5)}$

*Corresponding author: e-mail: ynchang@nfu.edu.tw 
Solar power consists of sunlight energy that is directly incident into the solar panel and is called directly incident solar power $P_{\mathrm{D}}$. Moreover, it also includes solar energy that penetrates the atmosphere and is scattered and diffused in random directions, socalled undisciplined power $P_{\mathrm{U}}$. Generally, the solar panel collecting power is directly related to the sunlight orientation, whereas the undisciplined power is not. The detailed solar power information of a solar energy sensor should include sunlight orientation, directly incident solar power, and solar undisciplined power.

Unlike the common solar power sensor, which combines directly incident solar power and solar undisciplined power together, in this research, we developed a solar power sensor possessing the capability to determine the proportion between directly incident solar power and solar undisciplined power. In the proposed solar power sensor, five photodiode detectors are installed on a rectangular pyramid column sensor. On each facet, a photodiode sensor is installed to collect solar light power coming from different orientations including the directly incident and undisciplined power. By applying a perpetual calendar to obtain the corresponding azimuth angle and elevation angle of sunlight, the research results can offer the solar energy industry and government energy authority with valuable solar power information.

\section{Configuration of Rectangular Pyramid Column Sensor}

Figure 1 shows the construction of the proposed rectangular pyramid column sensor. It comprises four trapezoid planes inclined $45^{\circ}$ with respect to the horizontal side and labeled east, west, south, and north. In each trapezoid plane facet, a photodiode detector is mounted. The collected powers on the east, west, south, north, and horizontal facets are designated as $P_{\mathrm{E}}, P_{\mathrm{W}}, P_{\mathrm{S}}, P_{\mathrm{N}}$, and $P_{\mathrm{H}}$, respectively. The directly incident angles are different and so are the collected solar power. In Fig. 1, as the sunlight comes from the north-east direction, the collected undisciplined powers of five photodiode detectors are very close. However, the west and south facet detectors can collect less directly incident light and absorb nearly zero directly incident power. Obviously, the collected power on the east detector $P_{\mathrm{E}}$ will be greater than that on the west detector $P_{\mathrm{W}}$, i.e., $P_{\mathrm{E}}>$ $P_{\mathrm{W}}$, similarly, $P_{\mathrm{N}}>P_{\mathrm{S}}$. The above description is based on the case that incident light is coming from the IV quadrant, as shown in Fig. 1. Similarly, this analysis can be applied to determine all the possible incident sunlight quadrants by comparing the photodiode power collected.

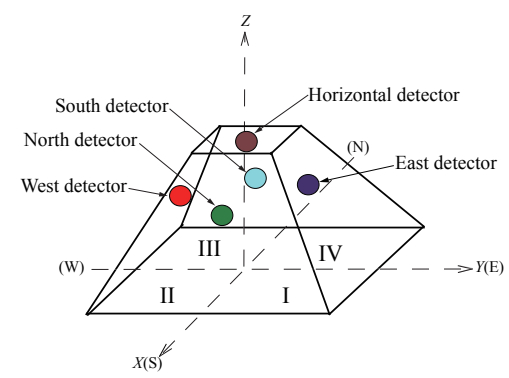

Fig. 1. (Color online) Model of rectangular pyramid column sensor for solar power measurement. 


\section{3D Coordinate and Polar Coordinate Representation}

Figure 2 demonstrates the 3D coordinate system, south is defined as the $X$-axis, east is defined as the $Y$-axis, and the normal vector direction is defined as the $Z$-axis. In definition, $r$ is the distance between the sun and the origin, $\alpha$ is the azimuth angle, $\varphi$ is the elevation angle, and the angle between the normal vector and the sunlight incident direction $\varphi_{\mathrm{i}}$ is the incident angle of sunlight. If the detector is located on the origin, the $3 \mathrm{D}$ coordinate of the sun is $(x, y, z)$. The relationship between $x, y, z$ and $r, \varphi, \alpha$ can be described as

$$
\begin{gathered}
x=r \cos \varphi \sin \alpha \\
y=r \cos \varphi \cos \alpha \\
z=r \sin \varphi .
\end{gathered}
$$

For a detector, if the incident angle $\varphi_{\mathrm{i}}$ can be derived from the perpetual calendar, the solar power collected by the detector can be expressed as

$$
\boldsymbol{P}_{\text {collected }}=\boldsymbol{P}_{\mathrm{D}} \times \cos \varphi_{\mathrm{i}}+\boldsymbol{P}_{\mathrm{U}} .
$$

Equation (2) reveals that the solar power collected by the detector, excluding undisciplined power, is proportional to $\cos \varphi_{\mathrm{i}}$.

In Fig. 1, the east and west planes can be regarded as the rotation of the horizontal plane along the $X$-axis. After rotating along the $X$-axis by $\theta_{x}$, the original position $(x, y, z)$ is transformed to position $\left(x^{\prime}, y^{\prime}, z^{\prime}\right)$. By rotating $\theta_{x}$ along the $X$-axis, $x^{\prime}, y^{\prime}$, and $z^{\prime}$ can be

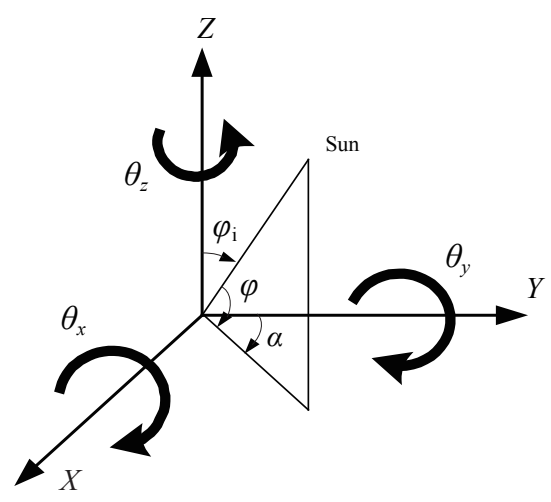

Fig. 2. $\varphi$ rotation along $X$-, $Y$-, and $Z$-axes. 
obtained as

$$
\left[\begin{array}{c}
x^{\prime} \\
y^{\prime} \\
z^{\prime}
\end{array}\right]=\left[\begin{array}{ccc}
1 & 0 & 0 \\
0 & \cos \theta_{x} & -\sin \theta_{x} \\
0 & \sin \theta_{x} & \cos \theta_{x}
\end{array}\right]\left[\begin{array}{l}
x \\
y \\
z
\end{array}\right]\left[\begin{array}{c}
\cos \varphi \sin \alpha \\
\cos \varphi \cos \alpha \cos \theta_{x}-\sin \varphi \sin \theta_{x} \\
\cos \varphi \cos \alpha \sin \theta_{x}+\sin \varphi \cos \theta_{x}
\end{array}\right] .
$$

The sunlight incident angle can be written as

$$
\theta_{i}=\cos ^{-1}\left(\cos \varphi \cos \alpha \sin \theta_{x}+\sin \varphi \cos \theta_{x}\right) .
$$

In Fig. 1, the south and north planes can be regarded as the rotation of the horizontal plane along the $Y$-axis. After rotating along the $Y$-axis by $\theta_{y}$, the original coordinate $(x, y$, $z)$ is transformed to coordinate $\left(x^{\prime}, y^{\prime}, z^{\prime}\right)$, and can be obtained as

$$
\left[\begin{array}{c}
x^{\prime} \\
y^{\prime} \\
z^{\prime}
\end{array}\right]=\left[\begin{array}{ccc}
\cos \theta_{y} & 0 & \sin \theta_{y} \\
0 & 1 & 0 \\
-\sin \theta_{y} & 0 & \cos \theta_{y}
\end{array}\right]\left[\begin{array}{l}
x \\
y \\
z
\end{array}\right]=r\left[\begin{array}{c}
\cos \varphi \cos \alpha \sin \theta_{y}+\sin \varphi \cos \theta_{y} \\
\cos \varphi \sin \alpha \\
-\cos \varphi \cos \alpha \cos \theta_{y}+\sin \varphi \sin \theta_{y}
\end{array}\right]
$$

The sunlight incident angle can be written as

$$
\theta_{i}=\cos ^{-1}\left(-\cos \varphi \sin \alpha \sin \theta_{y}+\sin \varphi \cos \theta_{y}\right) .
$$

Table 1 shows the formulas of incident angle $\varphi_{\mathrm{i}}$ in different facets. The east and west detectors are located on facets that rotate $\theta_{x}=45^{\circ}$ and $\theta_{x}=-45^{\circ}$ along the $X$-axis, respectively. The north and south detectors are located on facets that rotate $\theta_{y}=45^{\circ}$ and $\theta_{y}=-45^{\circ}$ along the $Y$-axis, respectively. Then, the incident angle of sunlight can be used to determine the incident quadrant based on the perpetual calendar. By using solar

Table 1

Formulas of incident angle $\varphi_{\mathrm{i}}$.

\begin{tabular}{lc}
\hline Facet & Incidence angle $\varphi_{\mathrm{i}}$ \\
\hline East & $\varphi_{\mathrm{E}}=\cos ^{-1}\left(\frac{\sqrt{2}}{2} \cos \varphi \cos \alpha+\frac{\sqrt{2}}{2} \sin \varphi\right)$ \\
West & $\varphi_{\mathrm{W}}=\cos ^{-1}\left(\frac{-\sqrt{2}}{2} \cos \varphi \cos \alpha+\frac{\sqrt{2}}{2} \sin \varphi\right)$ \\
North & $\varphi_{\mathrm{N}}=\cos ^{-1}\left(\frac{-\sqrt{2}}{2} \cos \varphi \cos \alpha+\frac{\sqrt{2}}{2} \sin \varphi\right)$ \\
South & $\varphi_{\mathrm{S}}=\cos ^{-1}\left(\frac{\sqrt{2}}{2} \cos \varphi \cos \alpha+\frac{\sqrt{2}}{2} \sin \varphi\right)$ \\
\hline
\end{tabular}


power data on the two detectors of incident quadrant, the directly incident solar power $P_{\mathrm{D}}$ and undisciplined solar power $P_{\mathrm{U}}$ can be obtained. In Table $2, P_{\mathrm{S}}, P_{\mathrm{W}}, P_{\mathrm{N}}, P_{\mathrm{E}}, \varphi_{\mathrm{S}}, \varphi_{\mathrm{W}}, \varphi_{\mathrm{N}}$, and $\varphi_{\mathrm{E}}$ can be achieved by applying detector power data and the perpetual calendar. $P_{\mathrm{D}}$ and $P_{\mathrm{U}}$ can be estimated on the basis of calculated quantities $\varphi_{\mathrm{S}}, \varphi_{\mathrm{W}}, \varphi_{\mathrm{N}}$, and $\varphi_{\mathrm{E}}$ as well as measured quantities $P_{\mathrm{S}}, P_{\mathrm{W}}, P_{\mathrm{N}}$, and $P_{\mathrm{E}}$.

\section{Experimental Results}

To verify the formulation, an experiment was performed on Jun 01, 2013. Table 3 shows the collecting solar power of five detectors and sunlight orientation. In Table 3, taking data at 8:00 A.M. as an example, the pyramid column sensor collected the incident light coming from the fourth quadrant; the detectors on the east and north facets can collect more power of directly incident solar power $P_{\mathrm{D}}$. The west facet can merely collect power of undisciplined incident solar light $P_{\mathrm{U}}$. Table 3 also shows the azimuth and elevation angles based on the perpetual calendar. $P_{\mathrm{D}}$ and $P_{\mathrm{U}}$ can be calculated using the formulas in Table 2. From 8:00 to 16:00, the solar power $P_{\text {solar }}$ is the sum of $P_{\mathrm{D}}$ and $P_{\mathrm{U}}$. Therefore, $P_{\mathrm{D}}, P_{\mathrm{U}}$, and $P_{\text {solar }}$ are listed in detail in Table 4.

Table 2

Formulas of solar power $P_{\mathrm{D}}$ and $P_{\mathrm{U}}$ in four sunlight incident quadrants.

\begin{tabular}{ccccc}
\hline Quadrant & I & II & III & IV \\
\hline Solar power & $\frac{P_{\mathrm{E}}-P_{\mathrm{S}}}{\cos \varphi_{\mathrm{E}}-\cos \varphi_{\mathrm{S}}}$ & $\frac{P_{\mathrm{S}}-P_{\mathrm{W}}}{\cos \varphi_{\mathrm{S}}-\cos \varphi_{\mathrm{W}}}$ & $\frac{P_{\mathrm{W}}-P_{\mathrm{N}}}{\cos \varphi_{\mathrm{W}}-\cos \varphi_{\mathrm{N}}}$ & $\frac{P_{\mathrm{N}}-P_{\mathrm{E}}}{\cos \varphi_{\mathrm{N}}-\cos \varphi_{\mathrm{E}}}$ \\
\hline$P_{\mathrm{D}}$ & $P_{\mathrm{E}}-P_{\mathrm{D}} \times \cos \varphi_{\mathrm{E}}$ & $P_{\mathrm{S}}-P_{\mathrm{D}} \times \cos \varphi_{\mathrm{S}}$ & $P_{\mathrm{W}}-P_{\mathrm{D}} \times \cos \varphi_{\mathrm{W}}$ & $P_{\mathrm{N}}-P_{\mathrm{D}} \times \cos \varphi_{\mathrm{S}}$ \\
\hline
\end{tabular}

Table 3

Elevation angle, azimuth angle, and solar power in five facets.

\begin{tabular}{lcccccccc}
$\begin{array}{c}\text { Facet } \\
\text { Time }\end{array}$ & $\begin{array}{c}P_{\mathrm{E}} \\
\left(\mathrm{W} / \mathrm{m}^{2}\right)\end{array}$ & $\begin{array}{c}P_{\mathrm{W}} \\
\left(\mathrm{W} / \mathrm{m}^{2}\right)\end{array}$ & $\begin{array}{c}P_{\mathrm{N}} \\
\left(\mathrm{W} / \mathrm{m}^{2}\right)\end{array}$ & $\begin{array}{c}P_{\mathrm{S}} \\
\left(\mathrm{W} / \mathrm{m}^{2}\right)\end{array}$ & $\begin{array}{c}P_{\mathrm{H}} \\
\left(\mathrm{W} / \mathrm{m}^{2}\right)\end{array}$ & $\begin{array}{c}\text { Elevation } \\
\text { angle }(\varphi)\end{array}$ & $\begin{array}{c}\text { Azimuth } \\
\text { angle }(\alpha)\end{array}$ & Quadrant \\
\hline $08: 00$ & 343.7 & 73.2 & 212.2 & 80.6 & 190.3 & $22.61^{\circ}$ & $344.86^{\circ}$ & IV \\
$09: 00$ & 533.7 & 93.8 & 338.5 & 103.1 & 358.1 & $35.99^{\circ}$ & $349.17^{\circ}$ & IV \\
$10: 00$ & 660.1 & 147.9 & 440.4 & 368.1 & 542.6 & $49.57^{\circ}$ & $353.28^{\circ}$ & IV \\
$11: 00$ & 726.0 & 300.4 & 533.3 & 503.8 & 681.0 & $3.25^{\circ}$ & $357.69^{\circ}$ & IV \\
$12: 00$ & 687.2 & 449.0 & 556.2 & 582.0 & 770.7 & $6.98^{\circ}$ & $4.44^{\circ}$ & I \\
$13: 00$ & 671.8 & 699.0 & 674.1 & 706.7 & 935.1 & $8.14^{\circ}$ & $118.95^{\circ}$ & II \\
$14: 00$ & 465.7 & 743.8 & 598.0 & 615.4 & 829.9 & $5.21^{\circ}$ & $176.86^{\circ}$ & II \\
$15: 00$ & 282.9 & 699.1 & 501.5 & 465.9 & 638.6 & $1.48^{\circ}$ & $182.98^{\circ}$ & III \\
$16: 00$ & 166.0 & 717.0 & 477.7 & 376.2 & 555.8 & $7.81^{\circ}$ & $187.31^{\circ}$ & III \\
\hline
\end{tabular}


Table 4

$P_{\mathrm{D}}, P_{\mathrm{U}}$, and $P_{\text {solar }}$ (unit: $\left.\mathrm{W} / \mathrm{m}^{2}\right)$.

\begin{tabular}{ccrc}
\hline Time & $P_{\mathrm{D}}$ & \multicolumn{1}{c}{$P_{\mathrm{U}}$} & $P_{\text {solar }}$ \\
\hline $08: 00$ & 286.2 & 85.6 & 371.8 \\
09:00 & 429.6 & 113.8 & 543.4 \\
10:00 & 546.8 & 116.8 & 663.6 \\
$11: 00$ & 631.4 & 126.5 & 757.9 \\
$12: 00$ & 718.4 & 78.2 & 796.6 \\
$13: 00$ & 862.1 & 80.1 & 942.2 \\
$14: 00$ & 753.8 & 92.6 & 846.4 \\
$15: 00$ & 618.3 & 106.5 & 724.8 \\
$16: 00$ & 582.7 & 137.2 & 719.9 \\
\hline
\end{tabular}

\section{Conclusions}

The proposed solar power sensor is supposed to collect the same undisciplined power on all detectors installed on five facets. The precision is far more reasonable under open space, such as grasslands, deserts, and shaded regions in the city. In this study, five photodetectors successfully constitute a rectangular pyramid column sensor by mounting them on the east, west, south, north, and horizontal facets therein. By further geometric transformation derivation, the directly incident solar power and undisciplined solar power can be obtained accordingly. By applying the perpetual calendar, the associated azimuth and elevation angles can be used to validate the accuracy of the proposed solar power sensor. Practically, the solar power sensor is realized and verified to possess an acceptable accuracy.

\section{Acknowledgements}

The authors would like to acknowledge the financial support from the National Science Council of Taiwan, through grant number NSC 102-2622-E-150-005-CC3.

\section{References}

1 O. Aliman and I. Daut: Proc. Int. Conf. POWERENG2007, 2007, pp. 551-555.

2 A. A. Khalil and M. El-Singaby: IEEE Proc. Int. Conf. 2003, pp. 1134-1137.

3 A. B. Maish: Photovoltaic Specialists Int. Conf. Record, 1990, pp. 864-869.

4 A. B. Maish, M. O'Neill, R. West and D. S. Shugar: IEEE Proc. Int. Conf, 1996, pp. 12111214.

5 D. V. Visa, A. Diaconescu and V. P. Duta: IEEE Proc. Int. Conf, 2008, pp. 449-454. 